GANIT J. Bangladesh Math. Soc. (ISSN 1606-3694) 32 (2012) 55-60

\title{
SINE-FUNCTION METHOD IN THE SOLITON SOLUTION OF NONLINEAR PARTIAL DIFFERENTIAL EQUATIONS
}

\author{
*M Abdur Rab and Jasmin Akhter \\ Department of Mathematics, Jahangirnagar University Savar Dhaka-1342 Bangladesh \\ *Email: nrab_math@yahoo.com
}

Received 15.05.2012 Accepted 03.11.2012

\begin{abstract}
In this paper we establish a traveling wave solution for nonlinear partial differential equations using sine-function method. The method is used to obtain the exact solutions for three different types of nonlinear partial differential equations like general equal width wave equation (GEWE), general regularized long wave equation (GRLW), general Korteweg-de Vries equation(GKDV) which are the important soliton equations.
\end{abstract}

Key words: GEWE, GRLW, GKDV, Sine-function method .

\section{Introduction}

The study of numerical methods for the solution of partial differential equations has been on intense activity over the last 40 years both from theoretical and practical point of view. Many of the partial differential equations arising from engineering and scientific applications were previously intractable. But since after improvements in numerical techniques along with the rapid advances in computer technology, those problems can now be routinely solved [1]. Differential operators are approximated in finite difference methods and thus difference equations are solved. In finite element method, the continuous domain is represented as a collection of a finite number of sub-domains known as elements. In the case of time-dependent problems, the differential equations are approximated by the finite element method to get a set of ordinary differential equations in time. These differential equations are solved approximately by finite difference method or some other ways. For all finite difference and finite elements it is necessary to have a boundary and initial conditions. But the Adomian decomposition method, developed by George Adomian, depends only on the initial conditions to obtain solution in series form which almost converges to the exact solutions of the problem. In recent years, some other ansatz methods have been developed such the tanh method [2,3], the extended tanhfunction method $[4,5]$, the modified extended tanh-function method [6-10] and the sinecosine method $[12,13]$.

Traveling waves are conspicuous in many linear and nonlinear modeling of wave, for example, sound wave, string wave, water wave etc. Many nonlinear models of wave can be found in different physical problems. Let us consider a simple example which admits traveling wave. Consider a one dimensional linear wave equation: $\frac{\partial^{2} u}{\partial t^{2}}=c^{2} \frac{\partial^{2} u}{\partial x^{2}}$, where $c$ denotes speed of the wave and $\mathrm{u}$ is a function of spatial variable $x$ and time variable $t$. 
The equation has the dôAlembert solution of the form

$$
u(x, t)=f(x-c t)+g(x+c t)
$$

The functions $f(x-c t)$ and $g(x+c t)$ describe right and left moving waves respectively with a constant speed $c$. The wave $f(x-c t)$ can be observed with a new system of co-ordinates $\left(x^{\prime}, t^{\prime}\right)$ so that $x=0$ at $t=0$. At time $t$, the position $x$ of the wave is $c t$, since $c$ is a speed of the wave, i.e. $x=c t$. Then the new co-ordinate is $(x-c t, t)$. This means the wave moves to the distance $(x-c t)$ at time $t$. Such waves arise in the study of nonlinear differential equations and waves are represented by the form $u(x, t)=f(x-c t)$, where $u(x, t)$ is a disturbance moving in the negative or positive $x$ direction if $c<0$ or $c>0$ respectively. If the traveling solution $u(x, t)$ depends only on the difference between the two coordinates of partial differential equations, then the solution keeps its shape exact. If the transition from the asymptotic state at $\xi=-\infty$ to the other asymptotic state at $\xi=\infty$ of a traveling wave is localized in $\xi$, where $\xi=x-c t$, then it is called solitary wave.

The objective of this paper is to use sine-function method to find the exact solutions of some nonlinear partial differential equations especially important soliton equations such as the general equal width wave (GEW) equation, the general regularized long wave (GRLW) equation, the general Korteweg-de Vries (GKDV) equation.

\section{The Sine-Function Method}

We consider the nonlinear partial differential equation in the form

$$
G\left(u, u_{t}, u_{x}, u_{x x}, u_{x x t}, \ldots . .\right)=0 \text {, }
$$

where $u(x, t)$ is the solution of the nonlinear partial differential equation (1).

Letô take the transformations,

$$
u(x, t)=f(\xi) \text { where } \xi=x-c t .
$$

Then we have the following transformations of differential operators :

$$
\frac{\partial}{\partial t}(\cdot)=-c \frac{d}{d \xi}(\cdot), \frac{\partial}{\partial x}(\cdot)=\frac{d}{d \xi}(\cdot), \quad \frac{\partial^{2}}{\partial x^{2}}(\cdot)=\frac{d^{2}}{d \xi^{2}}(\cdot), \quad \frac{\partial^{2}}{\partial x \partial t}(\cdot)=-c \frac{d^{2}}{d \xi^{2}}(\cdot) \ldots \ldots
$$

Using (3) into equation (1), we get

$$
G\left(f, f^{\prime}, f^{\prime \prime}, f^{\prime \prime \prime}, \ldots\right)=0
$$

Equation (4) is an ODE and can be integrated as many time as all terms contain derivatives and one can set integration constant to zero since $u(\xi), \frac{d u}{d \xi}, \frac{d^{2} u}{d \xi^{2}}, \frac{d^{3} u}{d \xi^{3}}, \ldots \ldots \rightarrow 0$ as $\xi \rightarrow \infty$ for the traveling wave.

Now the solution of equation (4) is set as 
$u(x, t)=f(\xi)=\lambda \sin ^{\beta}(\mu \xi), \quad|\xi| \leq \frac{\pi}{2 \mu}$,

where $\lambda, \beta, \mu$ are unknown parameters to be determined.

Then $f^{\prime}=\frac{d f(\xi)}{d \xi}=\lambda \beta \mu \sin ^{\beta-1}(\mu \xi) \cos (\mu \xi) \quad$ and

$f^{\prime \prime}=\frac{d^{2} f(\xi)}{d \xi^{2}}=-\lambda \beta \mu^{2} \sin ^{\beta}(\mu \xi)+\lambda \mu^{2} \beta(\beta-1) \sin ^{\beta-2}(\mu \xi)-\lambda \mu^{2} \beta(\beta-1) \sin ^{\beta}(\mu \xi)$

Equations (6) are substituted into equation (4) to give a trigonometric equation of $\sin ^{\alpha}(\mu \xi)$ terms. First we balance the exponents of each pair of sine to determine $\alpha$.

Then we collect all terms with the same power in $\sin ^{\beta}(\mu \xi)$ and put to zero their coefficients to get a system of algebraic equations among unknowns $\beta, \lambda$ and $\mu$. Therefore, the problem is reduced to a system of algebraic equations that can be solved for the unknowns using Mathematica. Once the unknown parameters are found, we obtain the solution as considered in (5) .

\section{Applications}

To exhibit the effectiveness of the proposed method three nonlinear partial differential equations are chosen and the method is applied there.

\subsection{The General Equal Width Wave Equation ( GEWE)}

We have the General Equal Width Wave Equation ( GEWE)

$$
u_{t}+\varepsilon u^{p} u_{x}-u_{x x t}=0
$$

By using the wave variable $\xi=x-c t$ and $u(x, t)=f(\xi)$, the GEWE is transformed into

$$
-c \frac{d f(\xi)}{d \xi}+\varepsilon f^{p}(\xi) \frac{d f(\xi)}{d}+c v \frac{d^{3} f(\xi)}{d \xi^{3}}=0
$$

Since all the terms contain derivatives, integrating once equation (8) gives,

$$
-c f(\xi)+\frac{\varepsilon}{p+1}(f(\xi))^{p+1}+c v \frac{d^{2} f(\xi)}{d \xi^{2}}=0
$$

Here we set the integration constant to zero, since $u(\xi), \frac{d u}{d \xi}, \frac{d^{2} u}{d \xi^{2}}$ etc. $\rightarrow 0$ when $\xi \rightarrow \infty$ and since this is a nonlinear traveling wave equation. 
Substituting equation (6) into equation (9), we get

$$
\begin{aligned}
& -c \lambda \sin ^{\beta}(\mu \xi)+\frac{\varepsilon \lambda^{p+1}}{p+1} \sin ^{\beta(p+1)}(\mu \xi)-c v \lambda \beta \mu^{2} \sin ^{\beta}(\mu \xi)+c v \lambda \mu^{2} \beta(\beta-1) \sin ^{\beta-2}(\mu \xi) \\
& -c v \lambda \mu^{2} \beta(\beta-1) \sin ^{\beta}(\mu \xi)=0
\end{aligned}
$$

Equating the exponents of sine functions and the coefficients,

$(p+1) \beta=\beta-2$,

$-c \lambda-c v \lambda \beta \mu^{2}-c v \lambda \mu^{2} \beta(\beta-1)=0$

$\frac{\varepsilon \lambda^{p+1}}{p+1}+c v \lambda \mu^{2} \beta(\beta-1)=0$

(11) is a system of algebraic equations.

Using Mathematica package for symbolic calculation, the system (11) is solved to obtain

$\beta=\frac{-2}{p}, \mu= \pm \frac{i p}{2 \sqrt{v}} \quad$ and $\quad \lambda=2^{-\frac{1}{p}}\left(\frac{c\left(2+3 p+p^{2}\right.}{\varepsilon}\right)^{\frac{1}{p}}$

Finally we substitute equation (12) into equation (5) and thereby obtain

$$
u(x, t)=2^{-\frac{1}{p}}\left(\frac{c\left(2+3 p+p^{2}\right.}{\varepsilon}\right)^{\frac{1}{p}} \sin ^{-\frac{2}{p}}\left(\frac{ \pm i p}{2 \sqrt{v}}(x-c t)\right)
$$

When $p=1$, the solution matches with the soliton solution of the EWE as in [14], when $p=2$, the solution is the soliton solution of MEWE equation as in [16] and also we can get the soliton solution of the GEWE equation as in [17]. It is to be mentioned here that the obtained results are in good agreement with those found in [15-17].

\subsection{The General Regularized Long Wave (GRLW) Equation}

We consider the general regularized long wave equation for long waves in shallow water as in [18-22]:

$$
u_{t}+u_{x}+\varepsilon u^{p} u_{x}-v u_{x x t}=0
$$

Using the transformation $u(x, t)=f(\xi)$ where $\xi=x-c t$, the equation (13) is transformed into the ODE

$$
-c \frac{d f(\xi)}{d \xi}+\frac{d f(\xi)}{d \xi}+\varepsilon f^{p}(\xi) \frac{d f(\xi)}{d}+c v \frac{d^{3} f(\xi)}{d \xi^{3}}=0
$$

Since all the terms in (14) contain derivatives, integrating it once, we get

$$
(1-c) f(\xi)+\frac{\varepsilon}{p+1}(f(\xi))^{p+1}+c v \frac{d^{2} f(\xi)}{d \xi^{2}}=0
$$


We substitute equation (6) into (15) to obtain

$$
\begin{aligned}
& (1-c) \lambda \sin ^{\beta}(\mu \xi)+\frac{\varepsilon \lambda^{p+1}}{p+1} \sin ^{\beta(p+1)}(\mu \xi)-c v \lambda \beta \mu^{2} \sin ^{\beta}(\mu \xi)+c v \lambda \mu^{2} \beta(\beta-1) \sin ^{\beta-2}(\mu \xi) \\
& -c v \lambda \mu^{2} \beta(\beta-1) \sin ^{\beta}(\mu \xi)=0
\end{aligned}
$$

By equating the exponents and the coefficients of each pair of the sine functions, we get the following system of algebraic equations :

$$
\begin{gathered}
(p+1) \beta=\beta-2 \\
(1-c) \lambda-c v \lambda \beta \mu^{2}-c v \lambda \mu^{2} \beta(\beta-1)=0 \\
\frac{\varepsilon \lambda^{p+1}}{p+1}+c v \lambda \mu^{2} \beta(\beta-1)=0
\end{gathered}
$$

Using Mathematica, we solve this system of equations and thereby obtain

$$
\beta=\frac{-2}{p}, \mu= \pm \frac{p \sqrt{1-c}}{2 \sqrt{v c}} \text {, and } \lambda=2^{-\frac{1}{p}}\left(\frac{-2+2 c-3 p+3 c p-p^{2}+c p^{2}}{\varepsilon}\right)^{\frac{1}{p}}
$$

Finally substituting equation (19) into equation (5), we get

$$
u(x, t)=2^{-\frac{1}{p}}\left(\frac{-2+2 c-3 p+3 c p-p^{2}+c p^{2}}{\varepsilon}\right)^{\frac{1}{p}} \sin ^{-\frac{2}{p}}\left( \pm \frac{p \sqrt{1-c}}{2 \sqrt{\nu c}}(x-c t)\right)
$$

which is the exact soliton solution of the GRLW equation .

\subsection{The General Korteweg-de Vries (GKDV)}

The equation ([23], [24]) for long waves in shallow water has the form:

$u_{t}+\varepsilon u^{p} u_{x}+\gamma u_{x x x}=0$

We follow the same procedures as applied into the previous two sections 3.1 and 3.2, and obtain the following system of equations :

$(p+1) \beta=\beta-2$

$$
-c \lambda-\gamma \lambda \beta \mu^{2}-\gamma \lambda \mu^{2} \beta(\beta-1)=0
$$

$\frac{\varepsilon \lambda^{p+1}}{p+1}+\gamma \lambda \mu^{2} \beta(\beta-1)=0$

Now we use Mathematica to solve this system of algebraic equations to obtain

$$
\beta=-\frac{2}{p}, \mu= \pm \frac{i p \sqrt{c}}{2 \sqrt{\gamma}}, \text { and } \lambda=2^{-\frac{1}{p}}\left(\frac{c\left(2+3 p+p^{2}\right)}{\varepsilon}\right)^{\frac{1}{p}}
$$


Substituting the equation (21) into equation (5), we get

$u(x, t)=2^{-\frac{1}{p}}\left(\frac{c\left(2+3 p+p^{2}\right)}{\varepsilon}\right)^{\frac{1}{p}} \sin ^{-\frac{2}{p}}\left( \pm \frac{i p \sqrt{c}}{2 \sqrt{\gamma}}(x-c t)\right)$

which is the exact soliton solution of GKDV .

\section{Conclusion}

In this paper, the sine-function method has been successfully applied to find the solution for three nonlinear partial differential equations such as GEWE, GRLW and GKDV equations. The sine-function method is used to find a new exact solution. Therefore, we can say the proposed method can be extended to solve the problems of nonlinear partial differential equations arising in the theory of solitons and other areas.

\section{REFERENCES}

[1] A. R. Mitchell, D.F. Griffiths, The Finite Difference Method in Partial Differential Equations, John Wiley \& Sons, 1980.

[2] E.J. Parkes, B.R. Duffy, Comput. Physics Communicatio. 98 (1998) 288

[3] A.H. Khater, W. Malfliet, D.K. Callebaut, E.S. Kamel, Chaos Solitons Fractals 14(2002) 513

[4] E. Fan, Physics Letter A 277 (2000) 212

[5] E. Fan, Z. Naturforsch, Physics Letter A 56 (2001) 312

[6] S. A. Elwakil, S.K. El-Labany, M.A. Zahran, R. Sabry, Physics Letter A 299 (2002) 179

[7] S. A. Elwakil, M.A. Abdou, Chaos Solitons Fractals 31 (2007) 840

[8] S. A. Elwakil, M.A. Abdou, Chaos Solitons Fractals 31 (2007) 1256

[9] A. A. Soliman. Physica A 361(2) (2006) 394

[10] M. A. Abdou, A.A. Soliman. Physics Letter A 353 (2006) 487

[11] M. A. Abdou, A.A. Soliman. J. Comput. Appl. Math. 181 (2) (2005) 245

[12] A. M. Wazwaz. Appl. Math. Comput. 161 (2) (2005) 561

[13] A. M. Wazwaz. Appl. Math. Comput. 161 (2) (2005) 575

[14] L. R. T. Gardner, G.A. Gardner, J. Comput. Phys. (1990) 218

[15] K.R. Raslan, Int. J. Comput. Math. 81(2004) 63

[16] D.J.Evans, K.R.Raslan, Int. J. Comput. Math. 81(4) (2005)445

[17] K.R.Raslan,J. Simulation Process Modeling 25(2004)

[18] J.C.Elibeck, G.R. McGuire, J. Comput. Phys. 23(1977)63

[19] L.R.T. Gardner, G.A. Gardner, J. Comput. Phys. 91 (1990) 441

[20] P.C. Jain, R. Shankar, T.V.Singh, Commun. Numer. Method Eng. 9(1993) 587

[21] L.R.T. Gardner, G.A. Gardner, A. Dogan, Commun. Numer. Methods Eng. 12(1996) 795

[22] S.I.Zaki, Comput. Phys. Commun. 138(2001)80

[23] A.H.A. Ali, Finite ElementStudies of the Korteweg-de Vries equation., Ph.D. Thesis, University College of North Wales, Bangor, 1989.

[24] Kh.O. Abdulloev, I.L.Bogolubsky, V.G. Makhankov, Phys. Letter. A 56 (6) (1976) 427. 